\title{
Low sun exposure increases multiple sclerosis risk both directly and indirectly
}

\author{
Anna Karin Hedström ${ }^{1} \oplus \cdot$ Tomas Olsson $^{1} \cdot$ Ingrid Kockum $^{1} \cdot$ Jan Hillert ${ }^{1} \cdot$ Lars Alfredsson $^{2,3}$
}

Received: 8 November 2019 / Revised: 10 December 2019 / Accepted: 12 December 2019 / Published online: 17 December 2019

(c) The Author(s) 2019

\begin{abstract}
Objective We aimed to study (1) to what extent the influence of low sun exposure on multiple sclerosis (MS) risk is mediated by low vitamin D levels; (2) whether low sun exposure or vitamin D deficiency act synergistically with HLA-DRB1*15:01 and absence of HLA-A*02:01.

Methods We used two population-based case-control studies (7069 cases, 6632 matched controls). Subjects with different HLA alleles, sun exposure habits and vitamin D status were compared regarding MS risk, by calculating odds ratios (OR) with 95\% confidence intervals (CI) employing logistic regression. Mediation analysis was used to identify the potential mediation effect of vitamin D on the relationship between low sun exposure and MS risk.

Results Low sun exposure increased MS risk directly as well as indirectly, by affecting vitamin D status. The direct effect, expressed as OR, was 1.26 (95\% CI 1.04-1.45) and the indirect effect, mediated by vitamin D deficiency, was 1.10 (95\% CI $1.02-1.23)$. Of the total effect, nearly $30 \%$ was mediated by vitamin $\mathrm{D}$ deficiency. There was a significant interaction between low sun exposure and vitamin D deficiency (attributable proportion due to interaction 0.3, 95\% CI 0.04-0.5) accounting for about $12 \%$ of the total effect. Further, both factors interacted with HLA-DRB1*15:01 to increase MS risk.

Interpretation Our findings indicate that low sun exposure acts both directly on MS risk as well as indirectly, by leading to low vitamin D levels. The protective effect of sun exposure thus seems to involve both vitamin D and non-vitamin D pathways, which is of relevance for prevention, in particular for those with a genetic susceptibility to MS.
\end{abstract}

Keywords Multiple sclerosis $\cdot$ Sun exposure $\cdot$ Vitamin D $\cdot$ Human leukocyte antigen $\cdot$ Interaction

\section{Introduction}

MS is a chronic immune-mediated disease of the central nervous system with underlying genetic and environmental factors. The geographic variations in MS occurrence [1], and the changes in MS risk that occur with migration [2], have at least partly been attributed to differences in sun exposure levels [1,

Electronic supplementary material The online version of this article (https://doi.org/10.1007/s00415-019-09677-3) contains supplementary material, which is available to authorized users.

Anna Karin Hedström

anna.hedstrom@ki.se

1 Department of Clinical Neuroscience, Karolinska Institutet, 17177 Stockholm, Sweden

2 Institute of Environmental Medicine, Karolinska Institutet, Stockholm, Sweden

3 Centre for Occupational and Environmental Medicine, Stockholm County Council, Stockholm, Sweden
2]. Several reviews have described consistent evidence of an association between both low sun exposure and vitamin D deficiency and increased MS risk [3, 4]. Exposure to sun leads to the release of a number of secondary mediators capable of suppressing cell-mediated immunity, and sun exposure also modulates immune responses by stimulating endogenous production of vitamin $\mathrm{D}[3,4]$.

Interactions have been demonstrated between several lifestyle and environmental risk factors for MS and presence of the strongest genetic risk factor, the HLA-DRB $1 * 15: 01$ allele [5-8]. A synergistic effect between vitamin D and DRB1*15:01 has been suggested [9], whereas we found no such interaction in a previous study comprising a relatively small case-control sample [10].

In the present study, based on two Swedish populationbased case-control studies, we aimed to investigate to what extent the influence of low sun exposure on MS risk is mediated by vitamin $\mathrm{D}$. We also aimed to study whether low sun exposure or vitamin D deficiency act synergistically with 
HLA-DRB1*15:01and absence of HLA-A*02:01 to increase MS risk.

\section{Methods}

\section{Study design and study participants}

Two population-based case-control studies on genetic and environmental factors for MS were used. One study was Genes and Environment in Multiple Sclerosis (GEMS) in which prevalent cases fulfilling the McDonald criteria [11] were identified from the Swedish National MS-registry [12]. One control per case was randomly selected from the national population register, matched for age, gender, and residential area at the time of disease onset. The study participants were recruited between November 2009 and November 2011.

The second study was Epidemiological Investigation of Multiple Sclerosis (EIMS) with a study group comprising the Swedish population aged 16-70 years. Incident cases of MS were recruited via neurology units in Swedish hospitals, including all university hospitals. Cases were diagnosed by a neurologist according to the McDonald criteria [11]. For each case, two controls were randomly selected from the national population register, matched by age in 5-year intervals, gender, and residential area. The study period was April 2005 to June 2015.

Patients in the Swedish MS registry who had previously been included in EIMS, were not included in GEMS. Randomly selected controls included in EIMS, were not used as controls in GEMS. Thus, there was no overlap of participants between the studies. The studies were approved by the Regional Ethical Review Board at Karolinska Institutet. All participants gave their informed consent to participate.

\section{Data collection}

In both studies, information regarding environmental exposures and lifestyle factors was collected using a standardised questionnaire. The response rate was $82 \%$ for cases and $66 \%$ for controls in GEMS, and $93 \%$ for cases and $73 \%$ for controls in EIMS. Those who could not specify their sun exposure habits were excluded. All participants who filled out the questionnaire were asked to provide blood samples. Those who did not donate blood were excluded in the present report. The number of study subjects in each study is presented in supplementary Table 1.

\section{Genotyping and measurement of vitamin D}

For all participants in both studies who donated blood, HLADRB1 and HLA-A alleles were determined at four-digit resolution. Genotyping was performed on the MS replication chip [13] which is based on an Illumina exome chip to which approximately 90,000 custom markers were added with extra high density in the HLA region and HLA alleles were then imputed with HLA*IMP:02 [14].

For EIMS participants recruited between 2005 and 2009 $(N=2464)$, vitamin D status was measured as levels of 25-hydroxy-vitamin D using a chemiluminescent immunoassay from Diasorin (Diasorin AB, Sundbyberg, Sweden) and a LIAISON $®$ instrument provided by Diasorin $A B$ with equimolar measurement of both 25-hydroxy-vitamin $\mathrm{D}_{2}$ and $\mathrm{D}_{3}$. The vitamin $\mathrm{D}$ analysis of the blood samples was performed in a single batch.

\section{Definition of sun exposure habits and vitamin D deficiency}

In GEMS, information on outdoor activity during summer and winter in different age periods $(0-9,10-19,20-29,30-39$, $40-49$ and $>50$ years) was used as a measure of sun exposure. The frequency of outdoor activity was reported on a four-point scale including 'a couple of hours per day', 'a couple of hours per week', 'a couple of hours per month', and 'more seldom'. We constructed an index for sun exposure habits during the 10 -year period prior to disease onset by adding the numbers from the four-point scales together, and thus acquired a value between 2 (the lowest exposure) and 8 (the highest exposure). Based on the 25th percentile among controls (six), sun exposure during the 10-year period prior to disease onset was dichotomized into high or low exposure.

We also dichotomized sun exposure during summer and winter, respectively, into high or low exposure defining high exposure as sun exposure 'a couple of hours per week' or 'a couple of hours per day' whereas less frequent sun exposure was defined as low sun exposure.

In EIMS, participants answered three questions regarding ultraviolet radiation (UVR) exposure during the 5-year period prior to inclusion in the study [11] (supplementary Table 2). Each answer alternative was reported on a fourpoint scale and we constructed an index by adding the numbers together and thus acquired a value between 3 (the lowest exposure) and 12 (the highest exposure). Sun exposure was dichotomized based on the 25 th percentile among controls (five). Vitamin D deficiency was defined as a value less than $50 \mathrm{nmol} / 1$.

\section{Statistical analysis}

The studies were analyzed separately. Subjects with different sun exposure habits, vitamin D status and DRB1*15:01 status were compared with regard to MS risk by calculating OR with 95\% CI using unconditional logistic regression models, with adjustment for the matching variables [15]. 
Trend tests for dose response relationships regarding degree of sun exposure/vitamin D levels and risk of MS were performed using continuous variables in a logistic regression model (index value between 2 and 8 in GEMS, and index value 3-12 in EIMS).

We categorized subjects based on amount of sun exposure (low/high) and vitamin D levels (low/high) and studied the influence of each factor in the absence of the other factor. When studying both factors simultaneously, causal mediation analysis was used to asses to what extent the relationship between low sun exposure and MS risk was mediated by vitamin D deficiency. The method allowed for exposure-mediator interaction. The causal effects were estimated on the OR scale and the CI were calculated using the delta method [16]. Further, a potential interaction on the additive scale was calculated between low sun exposure and vitamin D deficiency, using the attributable proportion due to interaction (AP) together with a $95 \%$ confidence interval.

The analyses were adjusted for age, gender, residential area, ancestry, smoking, adolescent BMI, infectious mononucleosis history, and the following MS-associated HLA alleles [17]; DRB1*03:01, DRB1*13:03, DRB1*08:01, B*44:02, B*38:01, B*55:01, DQA1*01:01, DQB1*03:02, DQB1*03:01, and homozygote correction for DRB 1*15:01, DRB $1 * 03: 01$ and $A * 02: 01$. When appropriate the analyses were also adjusted for DRB $1 * 15: 01, A^{*} 02: 01$ and sampling month.

Ancestry was dichotomized into Nordic versus non-Nordic origin. A participant who was born in any of the Nordic countries, whose parents had not immigrated from outside the Nordic countries, was classified as Nordic. Smoking was categorized into current, past or never smokers. Adolescent body mass index was calculated by dividing self-reported weight in kilograms by self-reported height in meters squared and dichotomized into more or less than $25 \mathrm{~kg} / \mathrm{m}^{2}$. A history of infectious mononucleosis was dichotomized into yes or no.

Adjustments were also made for a number of potential confounding variables that only had minor influence on the results. These factors were not kept in the final analyses and included skin reactivity, intake of vitamin supplements, intake of fatty fish, educational level, passive smoking, and alcohol consumption. Skin reactivity was categorized into the following groups based on how the subjects' skin react the first time they sunbathe in the summer without suntan lotion; 'I always go red, never suntanned', 'I always go red, sometimes suntanned', 'I sometimes go red, always suntanned', 'I never go red, get easily suntanned', and 'Always suntanned'. Intake of vitamin supplements was dichotomized into regular intake of multivitamins or vitamin $\mathrm{D}$ supplements or not. Intake of fatty fish was categorized into 'never', '1-3 times/months', and 'every week or daily'.
Educational level was categorized into no post-secondary education, post-secondary education without a university degree, or university degree. Passive smoking was dichotomized into ever or never exposed. Using the same cutoffs as those used by Statistics Sweden, alcohol consumption was categorized into the following groups based on the amount of alcohol intake per week at the index year: no consumption, low consumption $(<50 \mathrm{~g} /$ week for women and $<100 \mathrm{~g} /$ week for men), moderate consumption (50-112 g/week for women and 100-168 g/week for men), or high consumption ((>112 g/week for women and >168 g/week for men). All analyses were conducted using Statistical Analysis System (SAS) version 9.4.

\section{Results}

Our findings are based on 7069 cases and 6632 matched controls from two population-based case-control studies. The response rate was $82 \%$ for cases and $66 \%$ for controls in GEMS, and 93\% for cases and 73\% for controls in EIMS. The number of study subjects in each study is presented in supplementary Table 1 . In both studies, the median age at MS onset was 33 years. In GEMS, the median duration from the disease onset to inclusion in the study was 17 years. Almost all cases in the EIMS study were recruited within 1 year after the diagnosis and the questionnaires were completed after a median of 3.0 years following the onset of the disease. Characteristics of cases and controls, by low versus high sun exposure at the index year, are presented in supplementary Table 3.

\section{Sun exposure, vitamin D levels, and occurrence of MS}

In GEMS, low sun exposure both during summer and winter was associated with increased risk of MS (OR 1.3, 95\% CI 1.0-1.8 and OR 1.2, 95\% CI 1.0-1.5) (Table 1). In EIMS, low sun exposure during the 5-year period prior to study inclusion was associated with a $40 \%$ increased risk of MS (OR 1.4, 95\% CI 1.1-1.7). In both studies, there was a trend showing increasing occurrence of MS with decreasing sun exposure ( $p$ values for trend $<0.0001$ ).

In EIMS, there was a trend showing increased MS risk with decreasing vitamin D levels ( $p$ value for trend 0.0001 ). When both sun exposure and vitamin D levels were run in the same logistic regression model as continuous variables, both trends remained significant. The results also remained similar when the analyses were restricted to include only subjects of Nordic ancestry.

The trend showing increased MS risk with decreasing vitamin D levels were restricted to subjects with vitamin D levels below $50 \mathrm{nmol} / \mathrm{l}$. When the threshold of $50 \mathrm{nmol} / \mathrm{l}$ 
Table 1 OR with 95\% CI of MS for subjects with low sun exposure, compared to those with high sun exposure

\begin{tabular}{|c|c|c|c|c|c|c|}
\hline \multirow[t]{2}{*}{ Sun exposure (study) } & & \multicolumn{2}{|l|}{ Total } & \multicolumn{3}{|c|}{ HLA data available } \\
\hline & & $\mathrm{ca} / \mathrm{co}^{\mathrm{a}}$ & OR $(95 \% \mathrm{CI})^{\mathrm{b}}$ & $\mathrm{ca} / \mathrm{co}^{\mathrm{a}}$ & OR $(95 \% \mathrm{CI})^{\mathrm{b}}$ & OR $(95 \% \mathrm{CI})^{\mathrm{c}}$ \\
\hline \multicolumn{7}{|c|}{$\begin{array}{l}\text { Sun exposure during the } \\
10 \text {-year period prior to } \\
\text { disease onset (GEMS) }\end{array}$} \\
\hline Overall & $\begin{array}{l}\text { High } \\
\text { Low }\end{array}$ & $\begin{array}{l}5478 / 4923 \\
413 / 287\end{array}$ & $\begin{array}{l}1.0 \text { (reference) } \\
1.3(1.1-1.5)\end{array}$ & $\begin{array}{l}4673 / 3539 \\
339 / 210\end{array}$ & $\begin{array}{l}1.0 \text { (reference) } \\
1.2(1.0-1.5)\end{array}$ & $\begin{array}{l}1.0 \text { (reference) } \\
1.3(1.1-1.5)\end{array}$ \\
\hline Summer & $\begin{array}{l}\text { High } \\
\text { Low }\end{array}$ & $\begin{array}{l}5739 / 5122 \\
152 / 88\end{array}$ & $\begin{array}{l}1.0 \text { (reference) } \\
1.4(1.2-1.9)\end{array}$ & $\begin{array}{l}4889 / 3680 \\
123 / 69\end{array}$ & $\begin{array}{l}1.0 \text { (reference) } \\
1.3(1.0-1.8)\end{array}$ & $\begin{array}{l}1.0 \text { (reference) } \\
1.3(1.0-1.8)\end{array}$ \\
\hline Winter & $\begin{array}{l}\text { High } \\
\text { Low }\end{array}$ & $\begin{array}{l}5449 / 4890 \\
442 / 320\end{array}$ & $\begin{array}{l}1.0 \text { (reference) } \\
1.2(1.1-1.4)\end{array}$ & $\begin{array}{l}4646 / 3514 \\
336 / 235\end{array}$ & $\begin{array}{l}1.0 \text { (reference) } \\
1.2(1.0-1.4)\end{array}$ & $\begin{array}{l}1.0 \text { (reference) } \\
1.2(1.0-1.5)\end{array}$ \\
\hline \multicolumn{7}{|c|}{$\begin{array}{l}\text { Sun exposure during the } 5 \text {-year period } \\
\text { prior to study inclusion (EIMS) }\end{array}$} \\
\hline Overall & $\begin{array}{l}\text { High } \\
\text { Low }\end{array}$ & $\begin{array}{l}1749 / 4075 \\
1058 / 1869\end{array}$ & $\begin{array}{l}1.0 \text { (reference) } \\
1.3(1.2-1.5)\end{array}$ & $\begin{array}{l}1303 / 2006 \\
754 / 877\end{array}$ & $\begin{array}{l}1.0 \text { (reference) } \\
1.3(1.2-1.5)\end{array}$ & $\begin{array}{l}1.0 \text { (reference) } \\
1.4(1.2-1.6)\end{array}$ \\
\hline
\end{tabular}

${ }^{\mathrm{a} N u m b e r}$ of exposed cases and controls

${ }^{\mathrm{b}}$ Adjusted for gender, age, residential area, and ancestry

${ }^{c}$ adjusted for gender, age, residential area, ancestry, smoking, adolescent BMI, past IM, DRB1*15:01, DRB1*03:01, DRB1*13:03, DRB1*08:01, A*02:01, B*44:02, B*38:01, B*55:01, DQA1*01:01, DQB1*03:02, DQB1*03:01, and homozygote correction for DRB1*15:01, DRB $1 * 03: 01$ and $\mathrm{A} * 02: 01$

had been reached, vitamin D levels had no further significant impact on disease risk (supplementary Table 4).

\section{Causal mediation analysis in EIMS}

The mediation analysis was based on EIMS and restricted to subjects with data on vitamin D levels. The total effect of low sun exposure on MS risk, expressed as OR, was 1.38 (95\% CI 1.09-1.64). The direct effect was 1.26 (95\% CI $1.04-1.45)$ and the indirect effect, mediated by vitamin D deficiency, was 1.10 (95\% CI 1.02-1.23). Of the total effect, nearly $30 \%$ was mediated by vitamin $\mathrm{D}$ deficiency.

\section{Interaction between low sun exposure and vitamin D deficiency in EIMS}

Compared with subjects with high sun exposure without vitamin D deficiency, there was a synergistic effect between low sun exposure and vitamin $\mathrm{D}$ deficiency among doubleexposed subjects (AP 0.2, 95\% CI 0.04-0.5) (Table 2). Interaction between low sun exposure and vitamin D deficiency accounted for approximately $12 \%$ of the total effect.

\section{Interaction between low sun exposure, vitamin D deficiency, HLA-DRB1*15:01 and absence of HLA-A*02:01}

In both studies, DRB1*15:01 interacted with low sun exposure with regard to MS risk (AP 0.3, 95\% CI 0.1-0.6 in
Table 2 OR with $95 \%$ CI of developing MS among subjects with different combinations of sun exposure habits during the 5 -year period prior to study inclusion and vitamin D status

\begin{tabular}{llllll}
\hline Sun exposure & $\begin{array}{l}\text { Vitamin D } \\
\text { deficiency }\end{array}$ & $\mathrm{ca} / \mathrm{co}^{\mathrm{a}}$ & $\mathrm{OR}(95 \% \mathrm{CI})^{\mathrm{b}}$ & OR $(95 \% \mathrm{CI})^{\mathrm{c}}$ & $\mathrm{AP}(95 \% \mathrm{CI})$ \\
\hline High & No & $539 / 695$ & 1.0 (reference) & 1.0 (reference) & \\
High & Yes & $210 / 235$ & $1.1(0.9-1.4)$ & $1.2(1.0-1.6)$ & \\
Low & No & $174 / 220$ & $1.1(0.8-1.3)$ & $1.1(0.8-1.4)$ & \\
Low & Yes & $216 / 175$ & $1.6(1.3-2.0)$ & $1.7(1.3-2.1)$ & $0.2(0.04-0.5)$ \\
\hline
\end{tabular}

${ }^{a}$ Number of exposed cases and controls

${ }^{\mathrm{b}}$ Adjusted for age, gender, residential area, and ancestry

${ }^{\mathrm{c}}$ Adjusted for age, gender, residential area, ancestry, smoking, adolescent BMI, a history of IM, DRB1*03:01, DRB1*13:03, DRB1*08:01, A*02:01, B*44:02, B*38:01, B*55:01, DQA1*01:01, DQB1*03:02, DQB1*03:01, and homozygote correction for DRB1*15:01, DRB1*03:01 and A*02:01; AP attributable proportion due to interaction with $95 \%$ CI. The analysis is based on EIMS 
Table 3 OR with 95\% CI of MS among subjects with different combinations of DRB1*15:01 status and sun exposure habits during the 10-year period prior to disease onset

\begin{tabular}{|c|c|c|c|c|c|}
\hline DRB1*15:01 status & Overall sun exposure & $\mathrm{ca} / \mathrm{co}^{\mathrm{a}}$ & OR $(95 \% \mathrm{CI})^{\mathrm{b}}$ & OR $(95 \% \mathrm{CI})^{\mathrm{c}}$ & $\mathrm{AP}(95 \% \mathrm{CI})$ \\
\hline Negative & High & $1947 / 2523$ & 1.0 (reference) & 1.0 (reference) & \\
\hline Negative & Low & $135 / 157$ & $1.1(0.9-1.4)$ & $1.1(0.9-1.4)$ & \\
\hline Positive & High & $2726 / 1016$ & $3.5(3.2-3.9)$ & $3.7(3.3-4.1)$ & \\
\hline Positive & Low & $204 / 53$ & $5.1(3.7-6.9)$ & $5.9(4.3-8.1)$ & $0.3(0.14-0.6)$ \\
\hline DRB1*15:01 status & $\begin{array}{l}\text { Summer sun expo- } \\
\text { sure }\end{array}$ & $\mathrm{ca} / \mathrm{co}^{\mathrm{a}}$ & OR $(95 \% \mathrm{CI})^{\mathrm{b}}$ & OR $(95 \% \mathrm{CI})^{\mathrm{c}}$ & $\mathrm{AP}(95 \% \mathrm{CI})$ \\
\hline Negative & High & $2034 / 2629$ & 1.0 (reference) & 1.0 (reference) & \\
\hline Negative & Low & $48 / 51$ & $1.2(0.8-1.8)$ & $1.2(0.8-1.7)$ & \\
\hline Positive & High & $2855 / 1051$ & $3.6(3.3-3.9)$ & $3.8(3.4-4.2)$ & \\
\hline Positive & Low & $75 / 18$ & $5.4(3.2-9.0)$ & $6.0(3.5-10.1)$ & $0.3(0.01-0.7)$ \\
\hline DRB1*15:01 status & Winter sun exposure & $\mathrm{ca} / \mathrm{co}^{\mathrm{a}}$ & OR $(95 \% \mathrm{CI})^{\mathrm{b}}$ & OR $(95 \% \mathrm{CI})^{\mathrm{c}}$ & $\mathrm{AP}(95 \% \mathrm{CI})$ \\
\hline Negative & High & $1929 / 2508$ & 1.0 (reference) & 1.0 (reference) & \\
\hline Negative & Low & $153 / 172$ & $1.1(0.9-1.4)$ & $1.1(0.9-1.4)$ & \\
\hline Positive & High & $2717 / 1006$ & $3.6(3.3-3.9)$ & $3.7(3.4-4.2)$ & \\
\hline Positive & Low & $213 / 63$ & $4.5(3.4-6.0)$ & $5.2(3.8-7.0)$ & $0.3(0.03-0.5)$ \\
\hline
\end{tabular}

${ }^{a}$ Number of exposed cases and controls

${ }^{\mathrm{b}}$ Adjusted for age, gender, residential area, and ancestry

${ }^{\mathrm{c}}$ Adjusted for age, gender, residential area, ancestry, smoking, adolescent BMI, a history of IM, DRB1*03:01, DRB1*13:03, DRB1*08:01, A*02:01, B*44:02, B*38:01, B*55:01, DQA1*01:01, DQB1*03:02, DQB1*03:01, and homozygote correction for DRB1*15:01, DRB1*03:01 and $\mathrm{A}^{*} 02: 01 ; A P$ attributable proportion due to interaction with $95 \% \mathrm{CI}$. The analysis is based on GEMS

Table 4 OR with $95 \%$ CI of developing MS among subjects with different combinations of DRB1*15:01 and sun exposure habits during the 5 years prior to study inclusion

\begin{tabular}{llllll}
\hline DRB1*15:01 & Sun exposure & $\mathrm{ca} / \mathrm{co}^{\mathrm{a}}$ & OR $(95 \% \mathrm{CI})^{\mathrm{b}}$ & OR $(95 \% \mathrm{CI})^{\mathrm{c}}$ & AP $(95 \% \mathrm{CI})$ \\
\hline Negative & High & $577 / 1434$ & 1.0 (reference) & 1.0 (reference) & \\
Negative & Low & $358 / 649$ & $1.4(1.2-1.6)$ & $1.4(1.2-1.6)$ & \\
Positive & High & $726 / 572$ & $3.2(2.7-3.8)$ & $3.7(3.1-4.5)$ & \\
Positive & Low & $396 / 228$ & $4.4(3.6-5.4)$ & $5.3(4.2-6.6)$ & $0.2(0.1-0.4)$ \\
\hline
\end{tabular}

${ }^{a}$ Number of exposed cases and controls

${ }^{a}$ Adjusted for age, gender, residential area, and ancestry

${ }^{\mathrm{c}}$ Adjusted for age, gender, residential area, ancestry, smoking, adolescent BMI, a history of IM, DRB1*03:01, DRB1*13:03, DRB1*08:01, A*02:01, B*44:02, B*38:01, B*55:01, DQA1*01:01, DQB1*03:02, DQB1*03:01, and homozygote correction for DRB $1 * 15: 01, \mathrm{DRB} 1 * 03: 01$ and $\mathrm{A} * 02: 01 ; A P$ attributable proportion due to interaction with $95 \%$ CI. The analysis is based on EIMS
GEMS, and AP 0.2, 95\% CI 0.1-0.4 in EIMS) (Table 3-4). The interaction remained significant when sun exposure during summer and winter was analyzed separately (Table 4). In EIMS, an interaction was also observed between DRB1*15:01 and vitamin D deficiency at study inclusion (AP 0.2, 95\% CI 0.01-0.4) (Table 5). There was no interaction between low sun exposure or vitamin $\mathrm{D}$ deficiency and absence of $\mathrm{A} * 02: 01$ (data not shown).

\section{Discussion}

Our findings indicate that low sun exposure may increase MS risk directly as well as indirectly, by affecting vitamin D status. Of the total effect, nearly $30 \%$ was mediated by vitamin $\mathrm{D}$ deficiency. There was a significant interaction between low sun exposure and vitamin D deficiency, accounting for about $12 \%$ of the total effect. Our findings indicate that low sun exposure and vitamin D deficiency are different risk factors that act synergistically to increase MS risk.

In Sweden, there is insufficient UVR to produce vitamin D during winter [18], yet we observed that sun exposure both during summer and winter was associated with MS risk, which further supports the view that the protective effects of sun exposure involve both vitamin D and nonvitamin D pathways [3, 4]. Sun exposure is the principal source of vitamin $\mathrm{D}$ and evidence indicates a protective role for higher vitamin D levels [19, 20]. However, a multi-ethnic study observed that sun exposure reduced the risk of MS 
Table 5 OR with $95 \%$ CI of developing MS among subjects with different combinations of DRB1*15:01 and vitamin D deficiency

\begin{tabular}{llllll}
\hline DRB1*15:01 & $\begin{array}{l}\text { Vitamin D } \\
\text { deficiency }\end{array}$ & $\mathrm{ca} / \mathrm{co}^{\mathrm{a}}$ & OR $(95 \% \mathrm{CI})^{\mathrm{b}}$ & OR $(95 \% \mathrm{CI})^{\mathrm{c}}$ & AP $(95 \% \mathrm{CI})$ \\
\hline Negative & No & $307 / 660$ & 1.0 (reference) & 1.0 (reference) & \\
Negative & Yes & $208 / 311$ & $1.5(1.2-1.9)$ & $1.4(1.1-1.8)$ & \\
Positive & No & $406 / 255$ & $3.4(2.8-4.2)$ & $4.4(3.4-5.7)$ & \\
Positive & Yes & $218 / 99$ & $4.9(3.7-6.4)$ & $6.2(4.5-8.6)$ & $0.2(0.01-0.4)$ \\
\hline
\end{tabular}

${ }^{a}$ Number of exposed cases and controls

${ }^{\mathrm{a}}$ Adjusted for age, gender, residential area, and ancestry

${ }^{\mathrm{c}}$ Adjusted for age, gender, residential area, ancestry, smoking, adolescent BMI, a history of IM, DRB1*03:01, DRB1*13:03, DRB1*08:01, A*02:01, B*44:02, B*38:01, B*55:01, DQA1*01:01, DQB1*03:02, DQB1*03:01, and homozygote correction for DRB1*15:01, DRB1*03:01 and A*02:01; AP attributable proportion due to interaction with $95 \%$ CI. The analysis is based on EIMS regardless of race/ethnicity, whereas vitamin D deficiency was only associated with MS risk among whites [21]. Several studies have reported that sun exposure and vitamin D are independently associated with decreased MS risk [10, 22].

Apart from being essential for vitamin D production, sun exposure may result in suppression of cell mediated immunity, by the activation of regulatory $\mathrm{T}$ and $\mathrm{B}$ cells, and possibly by the range of cytokines and chemokines that are released following sun exposure [3, 23]. A suppressive effect of UVR through pathways independent of vitamin D have also been observed in experimental autoimmune enchephalomyelitis studies [24-26].

Both low sun exposure and vitamin D deficiency interacted with DRB1*15:01 to increase MS risk. Early studies have demonstrated that vitamin D influences HLA-DRB1 antigen expression and presentation [27]. There is a vitamin $D$ response element (VDRE) region in the promoter region of HLA-DRB1 which is highly conserved on DRB1*15:01 haplotypes, whereas the VDRE present on other HLA-DRB1 haplotypes is not responsive to 1,25-dihydroxyvitamin D3 [9]. The interaction between low sun exposure/vitamin D deficiency and DRB $1 * 15: 01$ indicates that these factors act on the immune system. The influence of several other lifestyle and environmental factors on MS risk is affected by the absence or presence of $A * 02: 01$ but this does not seem to be the case with sun exposure or vitamin $\mathrm{D}$. The more restricted interaction between low sun exposure/vitamin D deficiency and HLA class II molecules suggests a different immune related pathway.

Both studies were designed as case-control studies in which information regarding environmental exposures was collected retrospectively. There is a risk of recall bias, especially when asking about exposures that are established risk factors for developing the disease. The risk of recall bias is greater in GEMS which uses prevalent MS cases. In EIMS, recall bias was minimized by predominantly including cases of MS who had received their diagnosis within the past year. However, our observation of an association between sun exposure and MS risk was similar in GEMS and EIMS, and also in agreement with numerous previous studies.

MS may result in sun avoidant behavior because of sensitivity to heat [28] which could lead to an overestimation of the inverse association between sun exposure during the 5-year period before study inclusion and MS risk in EIMS. However, the results remained similar when only subjects with disease onset during the past 2 years were included. In GEMS, low sun exposure during the 10-year period prior to disease onset was considered to minimize the potential problem of reverse causation. Furthermore, all main results remained similar when we instead considered the influence of low sun exposure during the age period 10-19 years. However, few cases and controls reported low exposure during this period.

The Swedish MS registry, through which the GEMS cases were recruited, is part of the clinical documentation system and used in all Swedish neurology departments and has been estimated to include data on approximately $83 \%$ of the prevalent MS patients in Sweden [12]. The response rate with regard to participation in GEMS was $82 \%$ for cases and $66 \%$ for matched controls. The relatively low participation rate among controls could introduce selection bias. However, selection bias is probably modest since the prevalence of lifestyle factors, such as smoking and socioeconomic status, among the controls was consistent with that of the general population in similar ages [29]. Furthermore, there were no significant differences with respect to age, gender, or sun exposure habits between those who provided a blood sample and those who did not, indicating that selection bias did not take place in this step. We consider it unlikely that our findings would be affected by bias to a large extent, especially since such a bias would then depend both on HLA genotype and sun exposure habits.

In EIMS, a sun exposure index, ranging between 3 and 12 , was used as a measure of sun exposure. In our previous study on sun exposure and MS risk, based on EIMS, we used a higher cut-off (the median among controls) when defining high and low sun exposure habits, and we observed no synergistic effect between low sun exposure and DRB $1 * 15: 01$ 
with regard to MS risk [10]. In the present study, which has a larger number of cases and controls, low sun exposure was defined as a value below the 25 th percentile among controls. The results from the former study based on EIMS are thus not comparable with the results based on EIMS in the present study.

Vitamin D levels were measured after MS onset and these levels were assumed to reflect levels before disease onset. Therefore, we only show an association, without being able to confirm causality. However, our findings remained similar when we restricted the analysis to include only subjects with disease onset within the past year. The observed interaction between low sun exposure/vitamin D deficiency and DRB $1 * 15: 01$ also alleviates the magnitude of some potential biases in the interpretation of the influence from these lifestyle factors on MS risk, since DRB1*15:01 is not likely to determine exposure habits. We thus consider it unlikely that our findings would be affected by bias to a large extent, especially since such a bias would then depend on HLA types.

In the present study, we had the opportunity to take a large number of potential confounding factors into account, including skin reactivity to sun exposure, vitamin supplements, intake of fatty fish, smoking, education and genetics. The factors considered only had minor influence on the results. However, we cannot rule out that other factors related to sunexposure or the studied HLA genes partly could influence the magnitude or the estimated measure of interaction.

In conclusion, our findings indicate that low sun exposure may increase MS risk directly as well as indirectly, by affecting vitamin D status. We also found signs of interactions between both low sun exposure and vitamin D deficiency and DRB1*15:01 in relation to MS risk. The potential protective effect of sun exposure thus seems to involve both vitamin D and non-vitamin D pathways, which is of relevance for prevention, in particular for those with a genetic susceptibility to MS. Modest sun exposure habits could thus be recommended.

Acknowledgements Open access funding provided by Karolinska Institute.

Author contribution All authors contributed to study conception and design. AKH performed the statistical analyses and drafted the manuscript. All authors commented on previous versions of the manuscript. All authors read and approved the final manuscript.

Funding The study was supported by grants from the Swedish Medical Research Council; from the Swedish Research Council for Health, Working Life and Welfare, the Swedish Brain Foundation, Horizon2020 MultipleMS, and the Swedish Society for Medical Research.

\section{Compliance with ethical standards}

Conflicts of interest Hedström has nothing to disclose. Olsson reports grants from Swedish Research Council, grants from Knut and Alice Wallenberg Foundation, grants from Swedish Brain Foundation, dur- ing the conduct of the study; grants and personal fees from Biogen, grants and personal fees from Genzyme, grants and personal fees from Novartis, personal fees from Merck, outside the submitted work. Kockum received speaker's fees from Merck-Serono, and is involved in a project sponsored by Biogen. Hillert received honoraria for serving on advisory boards for Biogen and Novartis and speaker's fees from Biogen, Merck-Serono, Bayer-Schering, Teva and Sanofi-Aventis. He has served as principal investigator for projects sponsored by, or received unrestricted research support from, Biogen, Merck-Serono, TEVA, Novartis and Bayer-Schering. Alfredsson reports grants from Swedish Research Council, grants from Swedish Research Council for Health Working Life and Welfare, grants from Swedish Brain Foundation, during the conduct of the study; personal fees from Teva, personal fees from Biogene Idec, outside the submitted work.

Ethical standards All studies have been approved by the appropriate ethics committee and have, therefore, been performed in accordance with the ethical standards laid down in the 1964 Declaration of Helsinki and its later amendments. All participants in both studies provided their informed consent.

Open Access This article is licensed under a Creative Commons Attribution 4.0 International License, which permits use, sharing, adaptation, distribution and reproduction in any medium or format, as long as you give appropriate credit to the original author(s) and the source, provide a link to the Creative Commons licence, and indicate if changes were made. The images or other third party material in this article are included in the article's Creative Commons licence, unless indicated otherwise in a credit line to the material. If material is not included in the article's Creative Commons licence and your intended use is not permitted by statutory regulation or exceeds the permitted use, you will need to obtain permission directly from the copyright holder. To view a copy of this licence, visit http://creativecommons.org/licenses/by/4.0/.

\section{References}

1. Simpson S Jr, Wang W, Otahai P, Blizzard L, van der Mei IAF, Taylor BV (2019) Latutide continues to be significantly associated with the prevalence of multiple sclerosis: an updated metaanalysis. J Neurol Neurosurg Psychiatry 90:1193-1200

2. Rotstein DL, Marrie RA, Maxwell C, Gandhi S, Schults SE, Fung $\mathrm{K}, \mathrm{Tu} \mathrm{K}$ (2019) MS risk in immigrants in the McDonald era: a population-based study in Ontario, Canada. Neurology 93:e2203e2215. https://doi.org/10.1212/WNL.0000000000008611

3. Lucas RM, Byrne SN, Correale J, Ilschner S, Hart PHI (2015) Ultraviolet radiation, vitamin D and multiple sclerosis. Neurodegener Dis Manag 5:413-424

4. Sundström P, Salzer J (2015) Vitamin D and multiple sclerosis from epidemiology to prevention. Acta Neurol Scand 132:56-61

5. Xiao D, Ye X, Zhang N, Qu M et al (2015) A meta-analysis of interactions between Epstein-Barr virus and HLA-DRB1*15:01 on multiple sclerosis. Sci Rep 5:18083

6. Disanto G, Hall C, Lucas R, Ponsonby AL et al (2013) Assessing interactions between HLA-DRB $1 * 15$ and infectious mononucleosis on the risk of multiple sclerosis. Mult Scler 19:1355-1358

7. Hedström AK (2019) Smoking and its interaction with genetics in MS etiology. Mult Scler 25:180-186

8. Hedström AK, Lima Bomfim I, Barcellos L, Gianfrancesco M et al (2014) Interaction between adolescent obesity and HLA risk genes in the etiology of multiple sclerosis. Neurology 82:865-872

9. Ramagopalan SV, Maugeri NJ, Handunnetthi L, Lincoln MR et al (2009) Expression of the multiple sclerosis-associated MHC class 
II allele HLA-DRB1*1501 is regulated by vitamin D. PLoS One Genet 5:e1000369

10. Bäärnhielm M, Hedström AK, Kockum I, Sundqvist E et al (2012) Sunlight is associated with decreased multiple sclerosis risk: no interaction with human leukocyte antigen-DRB $1 * 15$. Eur J Neurol 19:955-962

11. Thompson AJ, Montalban X, Barkhof F, Brochet B et al (2000) Diagnostic criteria for primary progressive multiple sclerosis: a position paper. Ann Neurol 47:831-835

12. Hillert J, Stawiarz L (2015) The Swedish MS registry clinical support tool and scientific resource. Acta Neurol Scand Suppl 132:11-19

13. Internet-based information: https://www.biorxiv.org/content/early /2017/07/13/143933. Accessed 10 Nov 2018.

14. Dilthey AL, Moutsianas S, Shen J et al (2013) Multi-population classical HLA type imputation. PLoS Comput Biol 9:e1002877

15. Pearce N (2016) Analysis of matched case-control studies. BMJ 25:352-i969

16. Valeri L, VanderWeele TJ (2013) Mediation analysis allowing for exposure-mediator interactions and causal interpretation: theoretical assumptions and implementation with SAS and SPSS macros. Psychol Methods 18:137-150

17. Moutsianas L, Jostins L, Beecham AH, Dilthey AT et al (2015) Class II HLA interactions modulate genetic risk for multiple sclerosis. Nat Genet 47:1107-1113

18. Engelsen $\mathrm{O}(2010)$ The relationship between ultraviolet radiation exposure and vitamin D status. Nutrients 2:482-495

19. Simpson S Jr, der Mei IV, Taylor B (2018) The role of vitamin D in multiple sclerosis: biology and biochemistry, epidemiology and potential roles in treatment. Med Chem 14:129-143

20. Rhead B, Baarnhielm M, Gianfrancesco M, Mok A et al (2016) Mendelian randomization shows a causal effect of low vitamin D on multiple sclerosis risk. Neurol Genet 2:e97
21. Langer-Gould A, Lucas R, Xiang AH, Chen LH et al (2018) MS Sunshine study: sun exposure but not vitamin D is associated with multiple sclerosis risk in blacks and hispanics. Nutrients 10:268

22. Lucas RM, Ponsonby AL, Dear K, Valery PC et al (2011) Sun exposure and vitamin $\mathrm{D}$ are independent risk factors for CNS demyelination. Neurology 76:540-548

23. Breuer J, Schwab N, Schneider-Hohendorf T, Marziniak M et al (2014) Ultraviolet B light attenuates the systemic immune response in central nervous system autoimmunity. Ann Neurol 75:739-758

24. Wang Y, Marling SJ, Beaver EF, Seyerson KS, Deluca HF (2015) Uv light selectively inhibits spinal cord inflammation and demyelination in experimental autoimmune encephalomyelitis. Arch Biochem Biophys 567:75-82

25. Hart PH, Lucas RM, Booth DR, Carroll WM et al (2017) Narrowband uvb phototherapy for clinically isolated syndrome: a trial to deliver the benefits of vitamin D and other uvb-induced molecules. Front Immunol 8:3

26. Becklund BR, Severson KS, Vang SV, DeLuca HF (2010) Uv radiation suppresses experimental autoimmune encephalomyelitis independent of vitamin D production. Proc Natl Acad Sci USA 107:6418-6423

27. Rigby WF, Waugh M, Graziano RF (1990) Regulation of human monocyte HLA-DR and CD4 antigen expression, and antigen presentation by 1,25-dihydroxyvitamin D3. Blood 76:189-197

28. Simmons RD, Ponsonby AL, van der Mei IA, Sheridan P (2004) What affects your ms? Responses to an anonymous, internet-based epidemiological survey. Mult Scler 10:202-211

29. Internet based information, https://www.scb.se (2018). Accessed 10 Nov 2018. 